\title{
Yogurt enriched with a natural extract rich in PAF inhibitors and polyphenols Study design and rationale of a randomized, double blinded, placebo-controlled, parallel intervention study
}

\author{
Maria Detopoulou, Tzortzis Nomikos, Elizabeth Fragopoulou, Mary Yannakoulia, \\ Adamantini Kyriakou, Demosthenes Panagiotakos, Smaragdi Antonopoulou
}

Department Nutrition and Dietetics, School of Health Sciences and Education, Harokopio University, Athens, Greece

\begin{abstract}
Platelet Activating Factor (PAF) is a pro-inflammatory mediator of atherogenesis. Food enriched with PAF inhibitors may have potential cardioprotective properties. For this purpose, we created a yogurt enriched with PAF inhibitors derived from byproducts of olive oil industry, which was consumed by volunteers in order to investigate whether its consumption can affect favorable cardiovascular risk factors. Ninety two $(n=92)$ apparently healthy volunteers (35-65 years) were randomized into three groups based on age, sex and BMI. Volunteers in the Group A consumed a maximum of one plain yogurt every 2 weeks, volunteers in the Group B consumed a non-enriched yogurt daily and volunteers in the Group C ate an enriched yogurt daily. The intervention lasted eight weeks. Biological samples (blood, urine and faeces) were collected at the beginning, in the meantime and at the end of intervention. The article aims to present the rationale, the detailed methodology and the baseline characteristics of the three intervention groups.
\end{abstract}

KEY WORDS: Platelet-activating factor, yogurt, randomized controlled trial, cardiovascular diseases, olive oil, byproducts, platelets, thrombosis, inflammation, functional foods

Corresponding author:

Professor Smaragdi Antonopoulou

Department Nutrition and Dietetics, School of Health

Sciences and Education, Harokopio University,

70, El. Venizelou street, GR-17671, Athens, Greece

tel.: +30 2109549230, fax.: +302109577050

e-mail: antonop@hua.gr

\section{INTRODUCTION}

Cardiovascular diseases (CVD) represent the main cause of mortality globally ${ }^{1}$ and the research on their underlying biochemical mechanisms is crucial. Our research team has suggested a theory that indicates Platelet-Activating

Submission: 11.02.2019, Acceptance: 16.04.2019 
Factor (PAF) as a key player for the development of atherosclerotic plaques. ${ }^{2} \mathrm{PAF}$ is the first known phospholipid mediator and its chemical structure (1-O-alkyl-2-acetylsn-glyceryl-phosphatidylcholine), was elucidated by Professor Demopoulos in 1979. ${ }^{3}$ PAF is produced by most cells that participate in atherosclerosis progression. Its production is highly augmented under conditions (inflammation, oxidative stress, endothelial damage) that also favor atherosclerosis. The released PAF acts in an autacoid, paracrine or juxtacrine fashion in neighboring cells initiating a plethora of intracellular and extracellular events that ultimately damage endothelium and initiate atherosclerosis. ${ }^{4}$

The role of diet on the development and progression of CVD has long been studied, ${ }^{5}$ however, there is very limited evidence on the interactions between PAF and dietary compounds and their effect on CVD risk, especially in humans. Our team has already contributed to this area by identifying and chemically elucidating dietary inhibitors of PAF's synthesis and actions. ${ }^{6}$ We have especially focused on typical foods of the Mediterranean Diet, trying to understand whether the cardioprotective role of this dietary pattern could also be attributed to the presence of PAF inhibitors in these Mediterranean foods. ${ }^{7}$ Our studies reported the presence of PAF inhibitors in the lipid fractions of yogurt, honey, cheese, fish and wine..$^{8-11}$ We have also demonstrated the existence of strong PAF inhibitors in a polar lipid extract of olive oil (OOPLE), which also exerts antiatherogenic properties in animal models. ${ }^{12}$ The incorporation of this fraction in foods that are consumed on a daily basis could lead to the development of functional foods with anti-inflammatory and thus cardioprotective properties. A reasonable argue against the production of a food enriched in olive oil polar lipids is that the intake of such molecules can be achieved in reasonable amounts by the daily consumption of olive oil especially in the countries of the Mediterranean region. However, it is doubtful that the usual daily consumption of olive oil ensures the adequate consumption of its polar lipids, at least in amounts that exert anti-atherosclerotic effect. This hypothesis is supported by the results of a previous study of our team in hypercholesterolemic rabbits, which showed that OOPLE reduces atherosclerotic plaque formation much more efficiently than olive oil containing the same amount of OOPLE. ${ }^{13}$ On the other hand, olive oil is a rather expensive raw material for the extraction of OOPLE since large quantities of it should be extracted for the production of adequate quantities of the extract. Under this perspective, our team looked for alternative sources of this extract such as olive oil mill byproducts. The research for PAF inhibitors in the various stages of olive oil and seed oil extraction showed that these biologically active lipids (BAL) are mainly contained in the very wet olive cake remaining (WOC), while the most potent of them were structurally characterized. ${ }^{14}$ Moreover, WOC contains much higher concentrations of phenolic/polyphenolic molecules in comparison to olive oil. Thus, WOC constitutes a rich source of PAF inhibitors and biophenols, molecules with a wide range of biological activities.

According to a recent meta-analysis that includes epidemiological, prospective cohort and cross-sectional studies, daily yogurt consumption is inversely associated with metabolic syndrome components. ${ }^{15}$ Moreover, preliminary results have shown that the consumption of a functional yogurt, enriched with bioactive lipids (BAL) from WOC could attenuate atherosclerotic plaque formation, in cholesterol-fed rabbits. ${ }^{16}$ The promising outcomes of the animal studies allowed our team to proceed to human studies in order to evaluate if the daily consumption of such a yogurt could favorably modulate cardiovascular risk factors in apparently healthy adults aged 35-65 years old.

\section{STUDY DESIGN}

\section{Overall study design}

This was a randomized, three arm, double-blinded, placebo-controlled, parallel-group study designed to evaluate if the daily consumption of a low-fat plain yogurt, enriched with BAL could favorably modulate cardiovascular risk factors of apparently healthy volunteers aged 35-65 years old. The study took place at the Department of Nutrition and Dietetics of Harokopio University in Athens, Greece and was registered in ClinicalTrials.gov (NCT02259205).

\section{Bioethics}

This study was conducted according to the guidelines laid down in the Declaration of Helsinki (1989) of the World Medical Association and all procedures involving human subjects were approved by the Bioethics Committee of Harokopio University (40/30-10-2013). Written informed consent was obtained from all subjects before their inclusion in the study.

\section{Participants}

Eligible participants were apparently healthy adults aged 35-65 years old. Volunteers were recruited through word of mouth, electronic and paper media advertisements. Exclusion criteria were the history of cardiovascular or any other inflammatory disease, presence of cold or flu, pregnancy, acute respiratory infection, dental problems, renal/hepatic diseases and medical treatment. Prior to 
Yogurt enriched with a natural extract rich in PAF inhibitors and polyphenols

intervention all participants were informed about the aims and procedures of the study and signed an informed consent. Initially, 170 individuals responded to our call however only 92 met the inclusion criteria and signed the informed consent. The enrolled participants were randomized to one of the three groups of the study described below.

\section{Intervention}

Volunteers were randomly allocated (block-randomization) into three intervention groups. Participants in Group A were advised to continue their regular diet and consume a maximum of one plain yogurt every 2 weeks. Participants in Group B were advised to continue their regular diet and consume one plain, non-enriched yogurt, every day and those in group $C$ were advised to continue their regular diet and consume one BAL-enriched yogurt every day. The intervention lasted 8 weeks. Volunteers' adherence to the study protocol was assessed by two $24 \mathrm{~h}$ recalls every two weeks conducted by trained personnel. Blood and urine samples were collected at baseline, at 4th week and at 8th week of the intervention while faeces were collected at baseline and at the end of the intervention for the enumeration of selected gut bacteria. A flow chart of the protocol was presented in Figure 1.

\section{Primary and secondary outcomes of the study}

The primary outcome of this study is to determine whether the consumption of a yogurt rich in PAF antagonists and phenolic compounds can induce changes of platelet activity. Platelet activity is assessed by measuring

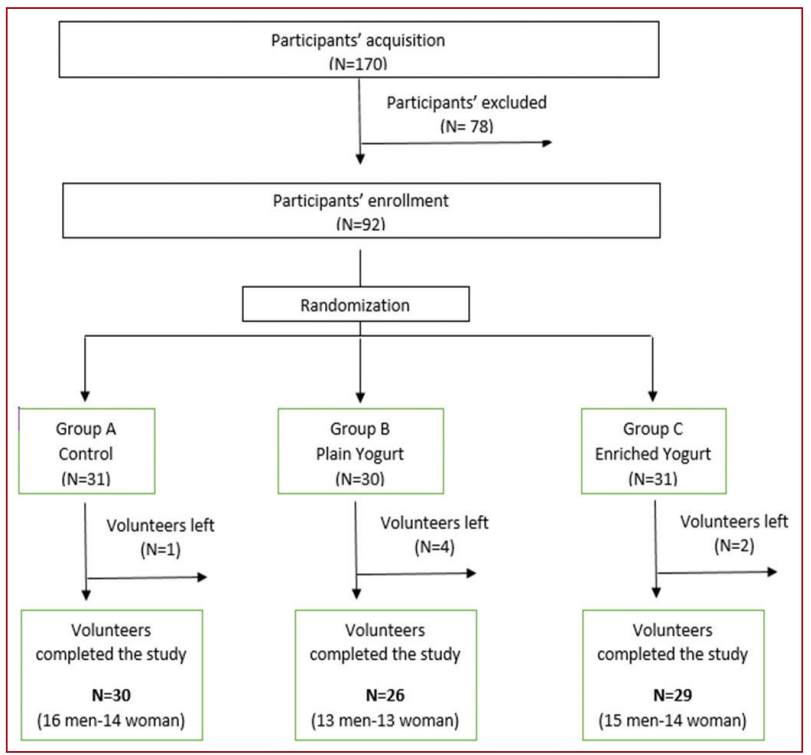

FIGURE 1. Flowchart of volunteers' recruitment. the ex vivo platelet sensitivity to classical in vivo activators of platelets, namely, PAF, ADP and thrombin (in the form of TRAP). Secondary outcomes of this study include changes in basic and novel cardiometabolic risk factors, such as indices of the glycemic (fasting glucose, insulin, HOMA-IR), lipidemic profile (triglycerides, LDL-C, HDL-C, total cholesterol), subclinical inflammation (CRP, TNFa, IL-6, IL-10), LpPLA 2 and dietary changes of the population (Figure 2).

\section{Safety monitoring}

All volunteers were questioned at follow-up visit and at $24 \mathrm{~h}$ telephone recalls for any potential side effects to the treatment, particularly gastrointestinal side effects. Gastrointestinal symptoms and possible side effects from yogurt consumption were assessed through validated questionnaires. ${ }^{17}$ Stool frequency and consistency of evacuations using the Bristol Stool Scale were also recorded. ${ }^{18}$

\section{Randomization}

Participants underwent block randomization according to gender, age and BMI. The distribution of participants in relation to the above variables was based on published data for the population of Greece, namely: a) in terms of the Body Mass Index, based on data from the epidemiological study ATTICA, ${ }^{19}$ b) in terms of gender, based on the 2011 national report, considering 50\% men and 50\% women in each group ${ }^{20}$ and c) in terms of age, it was also decided to allocate $50 \%$ between $35-51$ years old and 50\% between 51-65 years old.

\section{Production of the enriched yogurt}

Low fat plain yogurts (placebo) along with low-fat enriched yogurts were flavored with strawberry and were provided during the whole duration of the intervention by a Greek dairy industry (MEVGAL SA). The serving of both the plain and the enriched yoghurt was $150 \mathrm{~g}$. The yogurts, were produced by a pilot scale manufacturing line, and were physicochemically tested for their concentration in macronutrients and microbial parameters

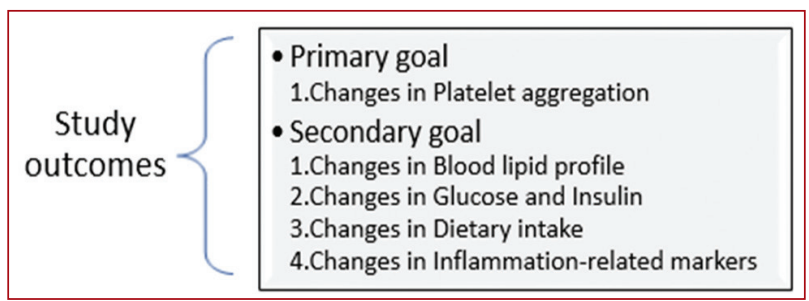

FIGURE 2. Study outcomes 
such as number of lactic acid bacteria, coliforms etc. The amount of enrichment with PAF inhibitors in yogurt was stable and was tested to be $0.6 \% \mathrm{w} / \mathrm{w}$. A chemical analysis of the two yoghurts was performed to determine the phenolic and biphenol components, three different time periods. The two yoghurts did not differ in the amount of these ingredients. In particular, plain yogurt contained $2.1 \pm 0.3 \mathrm{mg}$ phenolic compounds/g of lyophilized sample and $1.1 \pm 0.2 \mathrm{mg}$ of o-diphenolic compounds/g lyophilized sample while enriched yogurt contained $2.3 \pm 0.2 \mathrm{mg}$ phenolic compounds/g lyophilized sample and $0.8 \pm 0.1 \mathrm{mg}$ of o-diphenolic compounds/g lyophilized sample. The results presented are the mean values of experiments performed at three different time periods and no significant difference was detected between these time periods.

Various concentrations of enrichment with PAF inhibitors were tested. After organoleptic controls with trained volunteers it was found that $0.6 \%$ enrichment in yogurt was the highest acceptable.

The yogurts were delivered to volunteers in adequate amounts, on a weekly basis at pre-set dates to ensure their freshness. The composition of the plain and the enriched yogurt is shown in Table 1.

\section{Dietary and lifestyle evaluation of the volunteers}

Dietary intake of the participants at baseline and during the intervention were evaluated at a nutrient, food group and dietary pattern level using information from 24-h recalls and a food-frequency questionnaire. More particularly, three $24-\mathrm{h}$ recalls were collected at baseline, at 4 weeks and by the end ( 8 weeks) of the intervention period. The type of foods consumed (e.g., in terms of

TABLE 1. Composition of the plain and enriched yogurt

\begin{tabular}{lcc}
\hline & $\begin{array}{c}\text { Plain } \\
\text { Yoghurt }\end{array}$ & $\begin{array}{c}\text { Enriched } \\
\text { Yoghurt }\end{array}$ \\
\hline $\begin{array}{l}\text { Cow's skimmed milk and cream } \\
\text { and milk proteins }(\% \mathrm{w} / \mathrm{w})\end{array}$ & 84 & 83.4 \\
\hline Strawberry supplement $(\% \mathrm{w} / \mathrm{w})$ & 16 & 16 \\
\hline Polar lipid extract $(\% \mathrm{w} / \mathrm{w})$ & 0 & 0.6 \\
\hline Energy $(\mathrm{kJ} / \mathrm{kcal})$ & $356 / 85$ & $356 / 85$ \\
\hline Total Fat (g/100g) & 1.0 & 1.0 \\
Saturated Fat $(\mathrm{g} / 100 \mathrm{~g})$ & 0.6 & 0.6 \\
\hline Total Carbohydrate $(\mathrm{g} / 100 \mathrm{~g})$ & 14 & 14.2 \\
\hline Sugars (g/100g) & 13 & 13 \\
\hline Proteins (g/100g) & 5 & 5 \\
\hline Sodium (g/100g) & 0.1 & 0.1 \\
\hline
\end{tabular}

fat-content, brand name, constituents of mixed dishes, etc.), and the quantities or volumes were recorded in detail, using common household or other measures. Data from recalls were analyzed for their energy, macro- and micronutrient content by the Nutritionist Pro, version 2.2 software (Axxya Systems-Nutritionist Pro, Stafford, TX, USA), using a hand-coding procedure. The Nutritionist Pro food database was expanded by adding analyses of traditional Greek food and recipes. Adherence to the dietary intervention was evaluated on a regular basis by trained dietitians using 24-h recalls.

The food frequency questionnaire was validated for the Greek population. ${ }^{21}$ Responses were converted to daily or weekly intakes of specific food items and were grouped into food groups featuring the core foods of the traditional Greek diet. ${ }^{22}$ Adherence to the Mediterranean pattern was evaluated through the Mediterranean Dietary Score (MedDiet Score) proposed by Panagiotakos et al..$^{23}$ The scoring is based on the weekly consumption of eleven food groups and an individual score for each component is calculated, ranging from 0-5. The total MedDiet score ranges from 0 to 55, with higher values indicating greater adherence to the Mediterranean dietary pattern.

Baseline information about smoking habits, stress and socio-demographic conditions that may influence the investigated outcome were recorded through a standard questionnaire, ${ }^{24}$ that was developed for the purposes of the study. Physical activity was assessed with the use of a validated questionnaire (IPAQ-short version) and was expressed in metabolic equivalents minutes per week (MET-min-week). ${ }^{25}$

\section{Anthropometric and clinical measurements}

Weight and height were measured in light clothing, without shoes. Body mass index was then calculated as weight $(\mathrm{kg})$ divided by height ${ }^{2}\left(\mathrm{~m}^{2}\right)$. Waist circumference was measured between the superior iliac crest and the lower rib margin in the midaxillary line and hip circumference as the maximal horizontal circumference at the level of the buttocks. Both circumferences were measured to the nearest $0.1 \mathrm{~cm}$ and waist-to-hip ratio was calculated. Resting arterial blood pressure was measured three times in the right arm with subject in sitting position.

\section{Blood collection and isolation of biological samples}

Fasting venous blood $(50 \mathrm{~mL})$ was collected at baseline and at 4 and 8 weeks from the brachial vein and separated in EDTA and heparin-containing vacutainers, in adeninecitrate-dextrose polyethylene tube and in a vacutainer containing no anticoagulant. 
Yogurt enriched with a natural extract rich in PAF inhibitors and polyphenols

For the isolation of leukocyte homogenates 5 milliliters of heparinized blood were obtained from each volunteer. In order to induce erythrocyte sedimentation, $1.7 \mathrm{~mL}$ of dextran solution ( $3 \%$ dextran in $\mathrm{NaCl} 0.15 \mathrm{M}$ ) was added and the mixture was kept for $1 \mathrm{~h}$ at room temperature. The leukocyte rich supernatant was then centrifuged at $500 \times \mathrm{g}$ for $10 \mathrm{~min}$ at room temperature. Contaminating erythrocytes of the sediment were lysed with the addition of a lysis solution consisting of $155 \mathrm{mM} \mathrm{NH}_{4} \mathrm{Cl}, 10$ $\mathrm{mM} \mathrm{KHCO}_{3}$ and $0.1 \mathrm{mM}$ EDTA and then removed with a centrifugation at $300 \times \mathrm{g}$ for $10 \mathrm{~min}$ at room temperature. The pelleted cells were resuspended in $1 \mathrm{~mL}$ of a buffer containing $50 \mathrm{mM}$ Tris- $\mathrm{HCl}(\mathrm{pH}$ 7.4) and then sonicated on ice for 4 times of $10 \mathrm{sec}$ each. The leukocyte homogenate was aliquoted and stored at $-80^{\circ} \mathrm{C}$.

For the isolation of red blood cells (RBC) EDTA-anticoagulated blood was centrifuged at $1500 \times \mathrm{g}$ for $10 \mathrm{~min}$. The RBC pellet was washed 3 times with saline solution $(0.9 \%$ $\mathrm{NaCl}$ ) and packed $\mathrm{RBC}$ were stored at $-80^{\circ} \mathrm{C}$.

\section{Hematological and biochemical measurements}

Complete blood count was determined in EDTA anticoagulated whole blood with a Mindray BC-3000 hematology analyzer (Mindray, Shenzhen, P.R. China).

Serum glucose, triacylglycerols, total cholesterol, and HDL-C were determined enzymatically with an ACE biochemical analyzer (Schiapparelli Biosystems, Fairfield, NJ) using reagents from Alfa Wassermann (Woerden, the Netherlands). Serum insulin was determined by an immunoenzymometric assay on an AIA 600 II enzyme immunoassay system (Tosoh, Tokyo, Japan). LDL cholesterol was calculated by the Friedewald formula. ${ }^{26}$

CRP and IL-6, adiponectin and IL-10 were determined in serum by commercially available ELISA kits [IL-6 (IBL international), IL-10 (BioLegend), CRP, adiponectin and insulin (Invitrogen)].

\section{Ex vivo human platelet-rich plasma aggregation}

Blood $(10 \mathrm{~mL})$ was collected in polypropylene tubes containing $0.065 \mathrm{~mol} / \mathrm{L}$ citric acid and $0.085 \mathrm{~mol} / \mathrm{L}$ trisodium citrate as anticoagulant (blood to anticoagulant ratio of 9:1). Platelet-rich plasma (PRP) was obtained by centrifugation at $173 \mathrm{~g}$ for 8 minutes at $24^{\circ} \mathrm{C}$. Platelet-poor plasma (PPP) was obtained by further centrifugation of the residue at $1.120 \mathrm{~g}$ for 20 minutes at $24^{\circ} \mathrm{C}$. The concentration of platelets in the PRP was adjusted to 500.000 cells per microliter by adding appropriate amount of PPP to the PRP of the same donor.

The aggregation induced by various concentrations of PAF, TRAP and ADP was measured in human PRP. The maximum reversible or the least non reversible aggre- gation was evaluated for PAF and ADP to assess $100 \%$ aggregation. The plot of percentage aggregation (ranging from $20 \%$ to $80 \%$ ) vs different concentrations of the aggregatory agent was constructed. From this curve, the concentration of each aggregatory agent that induces $50 \%$ of maximum aggregation was calculated. This value was defined as the $\mathrm{EC}_{50}$, namely, equivalent concentration for $50 \%$ aggregation.

\section{Statistical analysis}

Statistical power analysis revealed that 25 participants in each arm was adequate to achieve statistical power equal to $83 \%$ at $5 \%$ significance level of two-sided hypotheses that evaluated 1 standard deviation (SD) difference of $\mathrm{EC}_{50}$ between groups.

Normality was tested with the Kolmogorov-Smirnov criterion. Normally distributed continuous variables are presented as mean values \pm standard deviation, while skewed variables as median and 25th-75th quartiles. Categorical variables were presented as relative frequencies (\%). Differences in the mean values of subjects' baseline characteristics between the different intervention groups were assessed by analysis of variance (ANOVA) after checking for homoscedasticity using the Levene test. Associations between categorical variables were evaluated using the $x^{2}$ test. If the variables were skewed the non-parametric Kruskal-Wallis test was performed. All reported P-values were based on two-sided tests and compared to a significance level of 5\%. SPSS 21 (SPSS Inc., Chicago, IL, USA) software was used for statistical analysis.

\section{Baseline characteristics of volunteers}

The baseline demographic, clinical, lifestyle, anthropometric, biochemical, hematological and platelet aggregation parameters of the volunteers are shown in Tables 2-5. No differences between groups were found for age, BMI, blood pressure, lipid profile (total cholesterol, HDL cholesterol, LDL cholesterol and triacylglyrerols) and glucose levels.

\section{DISCUSSION}

The current paper outlines the protocol for a randomized controlled trial aimed at evaluating the impact of a new functional enriched yogurt on various risk factors for atherothrombosis. Functional foods comprise the most dynamic sector of food industry, nowadays. Food scientists are interested in identifying, characterizing, and delivering bioactive agents (nutraceuticals) in foods designed to prevent or treat diseases. These "nutraceuticals" are claimed to have health benefits over and above their traditional role in human nutrition, including the ability to inhibit inflammation 
TABLE 2. Baseline characteristics of the study population

\begin{tabular}{|c|c|c|c|c|}
\hline & $\begin{array}{c}\text { Group A } \\
\text { (control group) }\end{array}$ & $\begin{array}{c}\text { Group B } \\
\text { (plain yogurt) }\end{array}$ & $\begin{array}{c}\text { Group C } \\
\text { (enriched yogurt) }\end{array}$ & $p$ \\
\hline Men/Women & $16 / 14$ & $13 / 13$ & $15 / 14$ & 0.75 \\
\hline Smoking (Yes/No) & $14 / 16$ & $12 / 13$ & $10 / 19$ & 0.19 \\
\hline Age (years) & $49.2 \pm 8.9$ & $48.8 \pm 9.2$ & $46.7 \pm 8.5$ & 0.53 \\
\hline Systolic blood pressure (mmHg) & $125.2 \pm 17.8$ & $128.7 \pm 17.3$ & $120.6 \pm 12.3$ & 0.17 \\
\hline Diastolic blood pressure $(\mathrm{mmHg})$ & $76.5 \pm 9.3$ & $77.4 \pm 9.2$ & $73.5 \pm 8.7$ & 0.24 \\
\hline BMI $\left(\mathrm{kg} / \mathrm{m}^{2}\right)$ & $27.7 \pm 6.4$ & $28.4 \pm 3.9$ & $27.2 \pm 3.6$ & 0.63 \\
\hline Glucose (mg/dL) & $95.3 \pm 18.2$ & $98.4 \pm 10.8$ & $99.9 \pm 30.6$ & 0.69 \\
\hline Triacylglycerols (mg/dL) & $112.8 \pm 59.0$ & $120.7 \pm 49.3$ & $115.1 \pm 50.5$ & 0.86 \\
\hline HDL-cholesterol (mg/dL) & $53.2 \pm 15.7$ & $54.0 \pm 12.6$ & $54.5 \pm 12.7$ & 0.97 \\
\hline LDL- cholesterol (mg/dL) & $134.4 \pm 35.6$ & $133.9 \pm 25.6$ & $141.8 \pm 32.2$ & 0.78 \\
\hline Insulin $(\mu \mathrm{U} / \mathrm{mL})$ & $12.1 \pm 8.4$ & $10.9 \pm 5.9$ & $11.5 \pm 8.2$ & 0.83 \\
\hline
\end{tabular}

Values are given as means+SDs apart from categorical variables which are given as absolute numbers.

TABLE 3. Baseline values of blood count parameters of the study population.

\begin{tabular}{lcccc}
\hline & $\begin{array}{c}\text { Group A } \\
\text { (control group) }\end{array}$ & $\begin{array}{c}\text { Group B } \\
\text { (plain yogurt) }\end{array}$ & $\begin{array}{c}\text { Group C } \\
\text { (enriched yogurt) }\end{array}$ & $p$ \\
\hline WBC $\left(10^{3} / \mu \mathrm{L}\right)$ & $6.74 \pm 1.78$ & $5.97 \pm 1.45$ & $6.17 \pm 1.74$ & 0.18 \\
RBC $\left(10^{6} / \mu \mathrm{L}\right)$ & $4.86 \pm 0.50$ & $4.68 \pm 0.41$ & $4.69 \pm 0.51$ & 0.22 \\
Hct $(\%)$ & $40.50 \pm 4.83$ & $40.48 \pm 4.73$ & $41.53 \pm 5.37$ & 0.28 \\
HGB $(\mathrm{g} / \mathrm{dL})$ & $13.75 \pm 1.75$ & $14.05 \pm 1.74$ & $14.31 \pm 1.92$ & 0.45 \\
Platelets $\left(10^{3} / \mathrm{L}\right)$ & $252 \pm 59$ & $260 \pm 72$ & $245 \pm 48$ & 0.97 \\
\hline
\end{tabular}

Values are given as means+SDs. WBC: White blood cells; RBC: Red blood cells; HGB: Hemoglobin; Hct: Hematocrit.

TABLE 4. Baseline $E_{50}$ values of PAF-, ADP- and TRAP-induced platelet aggregation

\begin{tabular}{lcccc}
\hline$(\mu \mathrm{M})$ & $\begin{array}{c}\text { Group A } \\
\text { (control group) }\end{array}$ & $\begin{array}{c}\text { Group B } \\
\text { (plain yogurt) }\end{array}$ & $\begin{array}{c}\text { Group C } \\
\text { (enriched yogurt) }\end{array}$ & $p$ \\
\hline PAF & $53.34(25.13-106.54)$ & $38.76(23.18-94.53)$ & $32.70(26.60-67.60)$ & 0.59 \\
ADP & $3.22(2.49-5.91)$ & $4.61(3.49-7.48)$ & $4.51(3.25-7.51)$ & 0.14 \\
TRAP & $0.86(0.70-0.99)$ & $0.89(0.67-1.14)$ & $0.90(0.70-1.27)$ & 0.76 \\
\hline
\end{tabular}

Values are given as median (25th-75th percent range). PAF: Platelet activating factor; ADP: Adenosine diphosphate; TRAP: Thrombin receptor activating peptide.

TABLE 5. Baseline values of subclinical inflammation markers

\begin{tabular}{lcccc}
\hline & $\begin{array}{c}\text { Group A } \\
\text { (control group) }\end{array}$ & $\begin{array}{c}\text { Group B } \\
\text { (plain yogurt) }\end{array}$ & $\begin{array}{c}\text { Group C } \\
\text { (enriched yogurt) }\end{array}$ & $p$ \\
\hline $\mathrm{IL}-6(\mathrm{pg} / \mathrm{mL})$ & $0.70(0.36-1.17)$ & $1.05(0.76-1.30)$ & $1.05(0.66-1.53)$ & $0.02 *$ \\
$\mathrm{IL}-10(\mathrm{pg} / \mathrm{mL})$ & $2.68(1.94-3.85)$ & $2.05(1.76-2.76)$ & $2.39(2.07-3.20)$ & $0.04 *$ \\
Adiponectin $(\mu \mathrm{g} / \mathrm{mL})$ & $5.74(3.61-8.51)$ & $3.85(2.62-5.43)$ & $4.49(2.51-7.48)$ & 0.21 \\
\hline
\end{tabular}

Values are given as median (25th-75th percent range). 
Yogurt enriched with a natural extract rich in PAF inhibitors and polyphenols

and cardiovascular diseases. Functional foods contain one or more nutraceutical agents dispersed within a natural or processed food product, such as fruit, vegetable, grain, seed, nut, beverage, and yogurt. In this attempt our group along with a well-known Greek dairy company try to incorporate bioactive ingredients (BAL) of the olives (WOC) into another typical food of the Mediterranean diet, namely yogurt. By this way we attempt to develop a nutraceutical with pleiotropic actions on cardiometabolic risk factors. It should also be mentioned that yogurt is the matrix which is primarily consumed, as a nutraceutical, from consumers. ${ }^{27}$

A major innovation of this project is that the raw material for the isolation of the bioactive extract is olive oil mill industry by products. Taking into account that the processing of olives results to an appreciable amount of by products, taking advantage of those by-products in a sustainable way is of primary importance for the olive oil producing countries. Many processes have been proposed for the recovery of the biologically active compounds of olive oil by products, many of them covered by patents. Using WOC as a source of anti-PAF and polyphenol extracts, which can be incorporated in functional foods, can render its management cost effective.

Our hypothesis concerning the health properties of the enriched yogurt is that the presence of anti-PAF molecules and phenolic compounds may provide a milieu of bioactive ingredients with proven anti-inflammatory, anti-platelet and antithrombotic properties. ${ }^{28}$ However, in contrast to most commercially available functional food which have health claims based only on in vitro and animal studies, our group wanted to proceed one step further to assess the efficacy of the functional yogurt by a randomized, dietary intervention study. We decided to conduct the study in a general, apparently healthy population for two reasons. Firstly, we would like to assess the acceptance of the product from a population of both sexes and a wide range of ages. Secondly, since this was the first intervention with this product, it was safer for us to estimate any side effects in a healthy population. In addition, the target market of this product was the general population, therefore we would like to investigate its health benefits to the group of consumers it was targeted in. The results of this study will guide us to further interventions with special populations.
The primary outcome of the study is platelet activity determined as ex vivo platelet sensitivity to plateletactivating agents. Apart from their well known role in atherothrombosis, ${ }^{29}$ subclinical activation of platelets can mediate the vascular complications of diabetic patients ${ }^{30}$ and be implicated in the pathogenesis of cancer and metastasis, Alzheimer's disease and immune dysregulation. ${ }^{31}$ For those reasons, a mild inhibition of platelet activity with dietary interventions and nutraceutical with antiplatelet properties can be proven protective against non-communicable diseases. In addition, the active ingredients of the enriched yogurt have shown strong antiplatelet properties in previous in vitro experiments. ${ }^{32}$ However, we cannot exclude the possibility that the enriched yogurt could favorably modulate the secondary outcomes of the study, especially subclinical inflammation, taking into account the strong anti-PAF actions of its constituents.

The baseline characteristics of the volunteers (Tables 2-5) indicate that our randomization ended up into three homogeneous groups concerning anthropometric, clinical, hematological and biochemical indices. There is a significant difference among the three groups in IL- 6 and IL-10 but the mean values of the variables indicate that those differences are clinically insignificant.

In conclusion, a novel yogurt enriched with a natural extract rich in PAF inhibitors and polyphenols was developed and its cardioprotective properties will be assessed after the conclusion of a dietary intervention study in general population. The rationale and study design of this intervention, which were described in this article, guarantee for the reliability of the outcomes of the study.

\section{Funding}

The study was co-funded by the European Regional Development Fund and National Resources, Code 11SYN_2_652, entitled "Cardioprotective properties of a yogurt enriched with bioactive lipids extracted from olive oil by products".

\section{Competing interests}

The study was investigator initiated and the dairy company had no involvement in the design and conduct of the study, in the collection, management analysis and interpretation of the data or in the preparation, review or approval of the manuscript. 


\title{
ПЕРІАНЧН
}

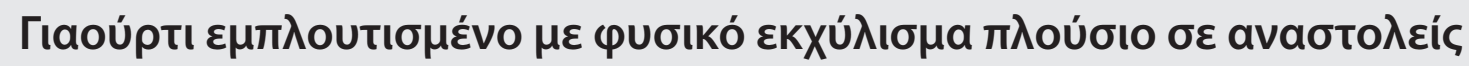

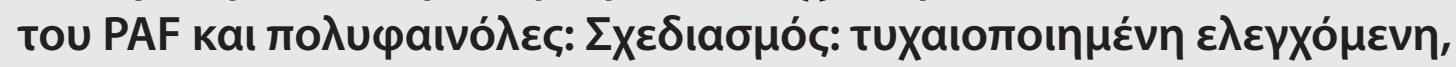

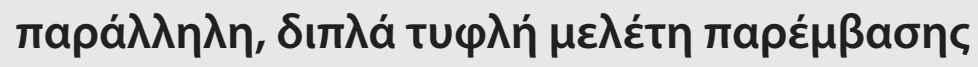

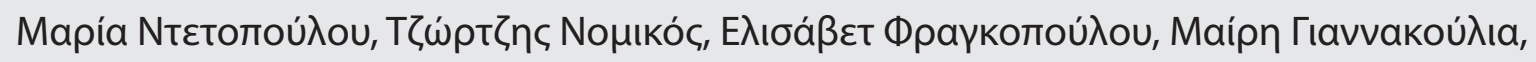

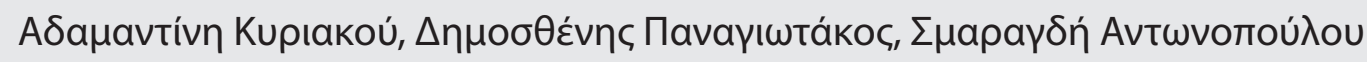

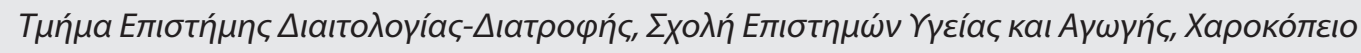

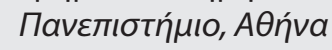

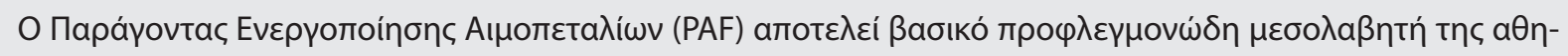

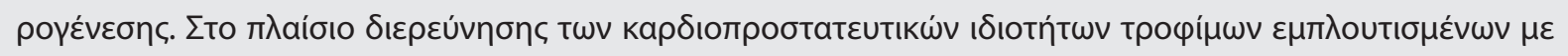

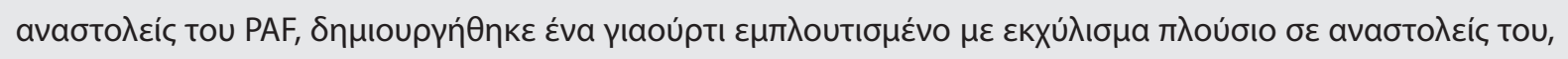

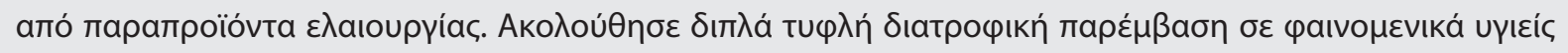

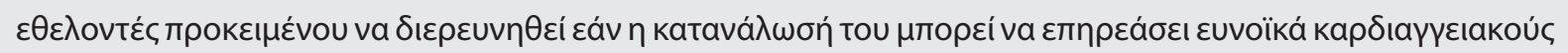

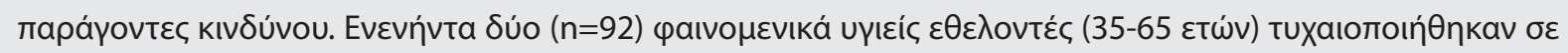



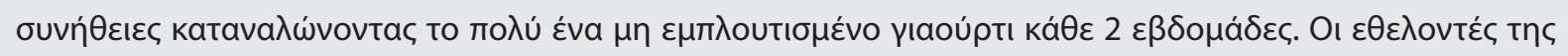

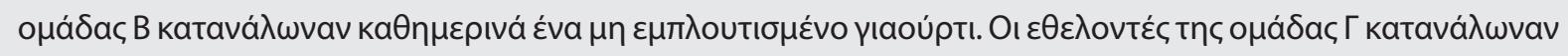

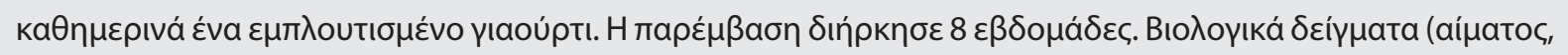

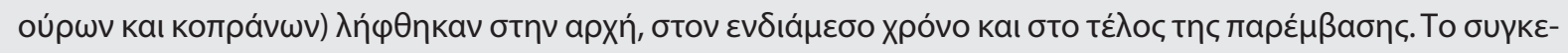

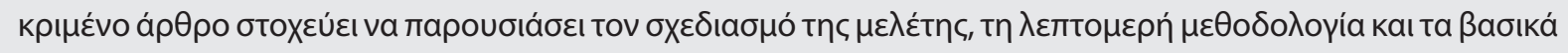

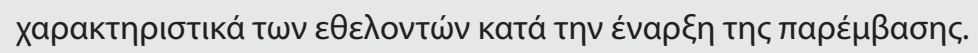

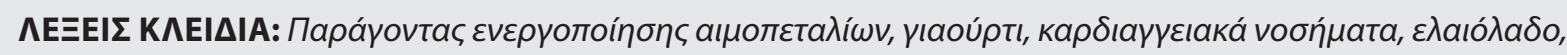

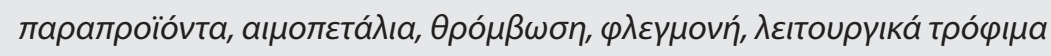

\section{REFERENCES}

1. Benjamin EJ, Virani SS, Callaway CW, Chamberlain AM, Chang AR, Cheng S, et al. Heart Disease and Stroke Statistics-2018 Update: A Report From the American Heart Association. Circulation. 2018 Mar;137(12):e67-e492.

2. Demopoulos CA, Karantonis HC, Antonopoulou S. Platelet activating factor - a molecular link between atherosclerosis theories. Eur J Lipid Sci Technol. 2003 Nov;105(11):70516.

3. Demopoulos CA, Pinckard RN, Hanahan DJ. Platelet-activating factor. Evidence for 1-O-alkyl-2-acetyl-sn-glyceryl3-phosphorylcholine as the active component (a new class of lipid chemical mediators). J Biol Chem. 1979 Oct;254(19):9355-8.

4. Antonopoulou S, Nomikos T, Karantonis HC, Fragopoulou E, Demopoulos CA. PAF, a potent lipid mediator. In: AT, editor. Bioactive phospholipids role in inflammation and atherosclerosis: Transworld Research Network, Kerala; 2008. p. 85-134.

5 Paoletti R, Poli A, Cignarella A. The emerging link between nutrition, inflammation and atherosclerosis. Expert Rev Cardiovasc Ther. 2006 May;4(3):385-93.

6. Nomikos T, Fragopoulou E, Antonopoulou S. Food Ingre- dients and Lipid Mediators. Curr Nutr Food Sci. 2007; 3(4):255-76.

7. Detopoulou P, Demopoulos CA, Karantonis HC, Antonopoulou S. Mediterranean diet and its protective mechanisms against cardiovascular disease: An insight into platelet activating factor (PAF) and diet interplay. Ann Nutr Disord Ther. 2015 Jan;2(1):1016.

8. Antonopoulou S, Semidalas CE, Koussissis S, Demopoulos CA. Platelet-Activating Factor (PAF) antagonists in foods: A study of lipids with PAF or anti-PAF-like activity in cow's milk and yogurt. J Agric Food Chem. 1996 Oct;44(10):304751.

9. Nasopoulou C, Smith T, Detopoulou M, Tsikrika C, Papaharisis L, Barkas D, et al. Structural elucidation of olive pomace fed sea bass (Dicentrarchus labrax) polar lipids with cardioprotective activities. Food Chem. 2014 Feb;145:1097-105.

10. Tsorotioti SE, Nasopoulou C, Detopoulou M, Sioriki E, Demopoulos CA, Zabetakis I. In vitro anti-atherogenic properties of traditional Greek cheese lipid fractions. Dairy Sci Technol. 2014 Mar;94(3):269-81.

11. Xanthopoulou MN, Kalathara K, Melachroinou S, Arampatzi-Menenakou K, Antonopoulou S, Yannakoulia M, et al. Wine consumption reduced postprandial platelet 
Yogurt enriched with a natural extract rich in PAF inhibitors and polyphenols

sensitivity against platelet activating factor in healthy men. Eur J Nutr. 2017 Jun;56(4):1485-92.

12. Antonopoulou S, Fragopoulou E, Karantonis HC, Mitsou E, Sitara M, Rementzis J, et al. Effect of traditional Greek Mediterranean meals on platelet aggregation in normal subjects and in patients with type 2 diabetes mellitus. J Med Food. 2006 Fall;9(3):356-62.

13. Karantonis HC, Antonopoulou S, Perrea DN, Sokolis DP, Theocharis SE, Kavantzas N, et al. In vivo antiatherogenic properties of olive oil and its constituent lipid classes in hyperlipidemic rabbits. Nutr Metab Cardiovasc Dis. 2006 Apr;16(3):174-85.

14. Karantonis HC, Tsantila N, Stamatakis G, Samiotaki M, Panayotou G, Antonopoulou S, et al. Bioactive polar lipdis in olive oil, pomace and waste byproducts J Food Biochem. 2008 Jul;32(4):443-59.

15. Lee $M$, Lee $H$, Kim J. Dairy food consumption is associated with a lower risk of the metabolic syndrome and its components: a systematic review and meta-analysis. $\mathrm{Br}$ J Nutr. 2018;120(4):373-84.

16. Stamatakis GM, Karantonis HC, Nasopoulou C, Gkogkaki V, Antonopoulou S, Perrea D, et al. Inhibition of atherogenesis in rabbits by yoghurt enriched with olive mill waste extracts. Hell J Atheroscler. 2012;3(3):212-2018.

17. Bovenschen $H J$, Janssen MJ, van Oijen MG, Laheij RJ, van Rossum LG, Jansen JB. Evaluation of a gastrointestinal symptoms questionnaire. Dig Dis Sci. 2006 Sep;51(9):150915.

18. Lewis SJ, Heaton KW. Stool form scale as a useful guide to intestinal transit time. Scand J Gastroenterol. 1997 Sep;32(9):920-4.

19. Panagiotakos DB, Pitsavos C, Chrysohoou C, Risvas G, Kontogianni MD, Zampelas A, et al. Epidemiology of overweight and obesity in a Greek adult population: the ATTICA Study. Obes Res. 2004 Dec;12(12):1914-20.

20. Hellenic Statistical Authority. Demographic and social characteristics of the Resident Population of Greece according to the 2011 Population - Housing Census. 2014. Available from: www.statistics.gr/en/2011-census-pophous

21. Bountziouka V, Bathrellou E, Giotopoulou A, Katsagoni C, Bonou M, Vallianou N, et al. Development, repeatability and validity regarding energy and macronutrient intake of a semi-quantitative food frequency questionnaire: methodological considerations. Nutr Metab Cardiovasc Dis. 2012 Aug;22(8):659-67.

22. Supreme_Scientific_Health_Council MoHaWoG. Dietary guidelines for adults in Greece. Arch Hellen Med. 1999;16:516-24.

23. Panagiotakos DB, Pitsavos C, Arvaniti F, Stefanadis C. Adherence to the Mediterranean food pattern predicts the prevalence of hypertension, hypercholesterolemia, diabetes and obesity, among healthy adults; the accuracy of the MedDietScore. Prev Med. 2007 Apr;44(4):335-40.

24. Panagiotakos DB, Pitsavos C, Stefanadis C. Dietary patterns: a Mediterranean diet score and its relation to clinical and biological markers of cardiovascular disease risk. Nutr Metab Cardiovasc Dis. 2006 Dec;16(8):559-68.

25. Hagströmer M, Oja P, Sjöström M. The International Physical Activity Questionnaire (IPAQ): a study of concurrent and construct validity. Public Health Nutr. 2006 Sep;9(6):75562.

26. Friedewald WT, Levy RI, Fredrickson DS. Estimation of the concentration of low-density lipoprotein cholesterol in plasma, without use of the preparative ultracentrifuge. Clin Chem. 1972 Jun;18(6):499-502.

27. Vella MN, Stratton LM, Sheeshka J, Duncan AM. Exploration of functional food consumption in older adults in relation to food matrices, bioactive ingredients, and health. J Nutr Gerontol Geriatr. 2013;32(2):122-44.

28. Parkinson L, Cicerale $S$. The health benefiting mechanisms of virgin olive oil phenolic compounds. Molecules. 2016 Dec;21(12).

29. Goto S, Hasebe T, Takagi S. Platelets: Small in size but essential in the regulation of vascular homeostasis translation from basic science to clinical medicine. Circ J. 2015;79(9):1871-81

30. Santilli F, Simeone P, Liani R, Davì G. Platelets and diabetes mellitus. Prostaglandins Other Lipid Mediat. 2015 Jul;120:28-39.

31. Ware J, Corken A, Khetpal R. Platelet function beyond hemostasis and thrombosis. Curr Opin Hematol. 2013 Sep;20(5):451-6.

32. Stamatakis G, Tsantila N, Samiotaki M, Panayotou GN, Dimopoulos AC, Halvadakis CP, et al. Detection and isolation of antiatherogenic and antioxidant substances present in olive mill wastes by a novel filtration system. J Agric Food Chem. 2009 Nov 25;57(22):10554-64. 